\title{
miR-100 resensitizes resistant epithelial ovarian cancer to cisplatin
}

\author{
PENG GUO $^{1,2}$, XIANGPENG XIONG ${ }^{2}$, SAINAN ZHANG ${ }^{2}$ and DONGXIAN PENG ${ }^{2}$ \\ ${ }^{1}$ Department of Obstetrics and Gynecology, Zhongshan People's Hospital, Zhongshan, Guangdong 528403; \\ ${ }^{2}$ Department of Obstetrics and Gynecology, Zhujiang Hospital, Southern Medical University, \\ Guangzhou, Guangdong 510515, P.R. China
}

Received May 4, 2016; Accepted September 5, 2016

DOI: $10.3892 /$ or. 2016.5140

\begin{abstract}
Epithelial ovarian cancer (EOC) is one of the malignant tumors that seriously affects women's health and chemotherapy resistance is an important reason for the poor prognosis. The present study was conducted to investigate whether microRNA-100 (miR-100) can be used to modulate the tolerance to cisplatin in EOC. Expression of miR-100 was compared between ovarian cancer cells tolerant and not tolerant to cisplatin. Mimic and antisense were used to study the roles and related mechanisms of miR-100 in cisplatin sensitivity in EOC. The alternation in the cisplatin sensitivity was investigated using grafted tumors derived from SKOV3/DDP cells with upregulated or downregulated miR-100 expression. miR-100 was lower in cisplatin resistant cell line SKOV3/DDP than in cisplatin sensitive cell line SKOV3. miR-100 might increase cisplatin sensitivity by inhibiting cell proliferation and conversion from G1 to S phase and increasing apoptosis. We showed that mTOR and PLK1 are targets of miR-100 and the cells were resensitized probably due to targeted downregulation of mTOR and PLK1 by miR-100. In vivo study with nude mice showed that tumors derived from miR-100 mimictransfected cells were more sensitive to cisplatin and had reduced expression of mTOR and PLK1. miR-100 resensitizes resistant epithelial ovarian cancer to cisplatin probably by inhibiting cell proliferation, inducing apoptosis and arresting cell cycle and by targeted downregulation of mTOR and PLK1 expression.
\end{abstract}

\section{Introduction}

Epithelial ovarian cancer (EOC) is one of the malignant tumors that seriously affects women's health. Because of insidious onset and rapid development, its mortality rate ranks the first among gynecological cancers (1). At present, cytoreductive surgery combined with platinum-based chemotherapy is effec-

Correspondence to: Dr Dongxian Peng, Department of Obstetrics and Gynecology, Zhujiang Hospital, Southern Medical University, 253 Industrial Avenue, Guangzhou, Guangdong 510515, P.R. China E-mail: pengdx8383@163.com

Key words: microRNA, ovarian cancer, cisplatin, drug resistance, mTOR, PLK1 tive in early stage of $70 \%$ of advanced stage patients, but the subsequent relapse and chemotherapy resistance often result in unimproved overall survival in ovarian cancer patients (2). The occurrence of chemotherapy resistance is an important reason for the poor prognosis $(3,4)$. Therefore, a better understanding of the mechanism underlying the drug resistance in ECO is essential to improve the poor prognosis.

The mechanisms of drug resistance to chemotherapy is very complex, and the possible mechanisms of cisplatin resistance include the pharmacokinetic factors (drug exposure and vascular tumor), micro-environmental factors (which are related to the impact on the cell cycle and apoptosis signaling) and cellular factors (such as the change in expression of proteins related to drug resistance, point mutations at drug target, enhanced cellular repair system and reduced apoptosis) (5-7). Genetic and epigenetic variations in the key genes in these pathways can result in drug resistance in cancer cells as well. A large number of studies have also demonstrated that micro-RNAs are playing regulatory role in the expression of these key genes $(8,9)$.

Micro-RNA (miRNA) is a group of endogenous and noncoding nucleotides of approximately 22 nucleotides long and functions through complementary pairing with 3'-untranslated region (3'-UTR) of target gene to regulate the expression of the target gene (10). Increasing evidence has demonstrated that the abnormal expression of miRNA is associated with the occurrence, development, metastasis and chemotherapy resistance of cancer (11). miR-100 is a miRNA identified earlier to be associated with tumor. It represses the post-transcription of mammalian target of rapamycin (mTOR) and Polo-like kinase 1 (PLK1) to control the growth, metastasis and chemotherapy sensitivity of tumor cells. Nagaraja et al (12) reported that in ovarian clear carcinoma cell line miR-100 is targeted to the (3'-UTR) of mTOR to suppress its expression, which results in increased sensitivity of the cell line to everolimus. Feng et al (13) found that in lung adenocarcinoma, the downregulation of miR-100 can activate target protein PLK1, thereby promoting taxol resistance (13). However, it is not clear whether miR-100 is associated with cisplatin resistance in EOC.

To explore the possible relationship between miR-100 expression and cisplatin resistance in EOC, we examined the expression of miR-100 in human ovarian cancer cell line SKOV3 and its cisplatin resistant cell line SKOV3/DDP. We upregulated and downregulated the expression of miR-100 in SKOV3/DDP to gain insights into the role of miR-100 in 
regulating apoptosis, proliferation, cell cycle and cisplatin sensitivity and in regulating the expression of mTOR and PLK1 in EOC. Mouse models were used to validate the resensitization of implanted cisplatin resistant tumor to cisplatin by miR-100. The findings will provide new strategies to overcome drug resistance.

\section{Materials and methods}

Cell lines and mice. Human ovary cancer cell line SKOV3 was a gift from the Cancer Research Institute of Southern Medical University; cisplatin resistant human ovary cancer cell line (SKOV/DDP) was a gift from the Central Laboratory, Qingdao University Medical College Affiliated Hospital. Female BALB/c-nu/nu nude mice (aged 4-5 weeks) were purchased from the Experimental Animal Center, Zhongshan University, under permit number SCKK (Guangdong) 20110029 and housed in the Animal Center on the North Campus of Zhongshan University. The animal experiments were approved by the Animal Care and Use Committee of Southern Medical University.

Cell culture. SKOV3 and SKOV3/DDP cells were cultured in RPMI-1640 containing $10 \%$ fetal bovine serum (FBS) at $37^{\circ} \mathrm{C}$ and $5 \% \mathrm{CO}_{2}$ in incubator with saturated humidity.

Cell transfection. SKOV3/DDP cells were transfected with lentivirus containing miR-100 mimics, (LV-miR-100), antisense (LV-anti-100) and empty vector (LV-NC) (Shanghai GenePharma, Co., Ltd., Shanghai, China). After 12 h, virus solution was removed and the cells were cultured in RPMI-1640 containing $10 \%$ FBS for another $48 \mathrm{~h}$ and examined for infection efficiency using a fluorescence microscope, and further confirmed using real-time quantitative PCR (qRT-PCR). The cells were cultured in complete culture medium containing puromycin $(2 \mu \mathrm{g} / \mathrm{ml})$ for two weeks to obtain SKOV3/DDP cells with miR-100 stably downregulated or upregulated.

$q R T-P C R$. RNA (3 $\mu \mathrm{g})$ was reverse transcribed using random primers (Applied Biosystems, Carlsbad, CA, USA). miRNA expression was determined using TaqMan ${ }^{\circledR}$ miRNA assays (Applied Biosystems) according to the manufacturer's protocol. In brief, $50 \mathrm{ng}$ of total RNA was reverse transcribed using specific miRNA primers. U6 expression was used for normalization using the comparative CT method (14). The primers (Shanghai GenePharma) were forward, 5'-ATCATTAAAC CCGTAGATCCGAA and reverse, 5'-GGAACGCTTCACGA ATTTG for miR-100; and forward, 5'-ATTGGAACGATACA GAGAAGATT and reverse, 5'-GGAACGCTTCACGAATT TG for U6.

Cell counting assay. Transfected SKOV3/DDP cells were seeded in wells of 96 -well plates at a density of $3 \times 10^{3}$ cells/well, cultured overnight and added with different concentrations of cisplatin from 0 (control) to $64 \mu \mathrm{g} / \mathrm{ml}$. For each concentration, 5-wells were used. The cells were cultured for $48 \mathrm{~h}$, added with $10 \mu \mathrm{l}$ CCK-8 solution (Dojindo Laboratories, Kumamoto, Japan) and cultured for another $4 \mathrm{~h}$ to measure the absorbance (A) at $450 \mathrm{~nm}$. The values were used to calculate the $\mathrm{IC}_{50}$ of cisplatin. In addition, the transfected SKOV3/DDP cells were seeded in wells of 96-well plates at a density of $2 \times 10^{3}$ and measured for absorbance at $450 \mathrm{~nm}$ wavelength at different time-points (12-72 h) using a plate reader to plot growth curves.

Western blot analysis. Total protein was extracted $72 \mathrm{~h}$ after transfection, separated on SDS-PAGE, transferred to membrane and incubated with primary antibodies (rabbit anti-human PLK1, anti-human mTOR and anti-human GAPDH monoclonal antibodies; Cell Signaling Technology, Beverly, MA, USA) for $1 \mathrm{~h}$, and secondary antibody (goat anti-rabbit IgG; Abcam, Cambridge, UK) for $1 \mathrm{~h}$. The membrane was developed using enhanced chemiluminescence (ECL) kit (Pierce, Rockford, IL, USA) and the gray value of the bands was quantified using Quantity One software to calculate the relative expression of mTOR and PLK1. The experiments were repeated 3 times.

FACS analysis. SKOV3/DDP cells with stably upregulated and downregulated miR-100 expression and control cells were cultured for $48 \mathrm{~h}$, harvested and stained with FITC Annexin V and propidium iodide (PI) (FITC Annexin V apoptosis detection kit; BD Biosciences, San Jose, CA, USA) for cell cycle analysis, they were fixed in $70 \%$ ethanol on ice, washed with phosphate-buffered saline (PBS), then stained with PI containing RNase A ( $20 \mu \mathrm{g} / \mathrm{ml}$ in PBS). The cells were analyzed on FACSVerse flow cytometry (BD Biosciences).

Crystal violet staining. Cells were inoculated in wells of 6 -well plates at a density of $1 \times 10^{2}$ cells/well and cultured for 14 days in RPMI-1640 medium with $10 \%$ FBS, fixed in methanol fixed, stained with $0.1 \%$ crystal violet solution. Samples were photographed and counted for the number of visible colonies.

Allograft and immunohistochemistry. Cells in logarithmic growth were adjusted to a cell density of $5 \times 10^{7} / \mathrm{ml}$ and injected subcutaneously into the nude mice at $200 \mu \mathrm{l} /$ mouse. When the tumors grew to $5 \mathrm{~mm}$ in diameter cisplatin was injected at $4 \mathrm{mg} / \mathrm{kg}$ every 3 days for a total of 7 times. The size of tumor was measured every 3 days to draw the growth curve. Three days after the end of treatment, the mice were sacrificed, tumor tissue removed, fixed in $10 \%$ formalin and used in immunohistochemistry assays for PLK1 and mTOR.

Statistical analyses. The SPSS 20 software was used for statistical analysis, measurement data were expressed as mean \pm standard derivation (SD). Paired groups were analyzed using Student's t-test, and one-way analysis of variance (ANOVA) was applied to compare the difference among multiple groups. Values with $\mathrm{P}<0.05$ was considered statistically significant.

\section{Results}

Resistance of SKOV3/DDP cells to cisplatin. We first determined the $\mathrm{IC}_{50}$ to measure the resistance to cisplatin. For SKOV3/DDP and SKOV3 cells, $\mathrm{IC}_{50}$ was 8.29 and $3.72 \mu \mathrm{g} / \mathrm{ml}$, respectively. Therefore, SKOV3/DDP was 2.23 times more resistant to cisplatin than SKOV3 (Fig. 1A).

Expression of miR-100. We then profiled the expression of miR-100 in the two cell lines. It was found that the expression of 


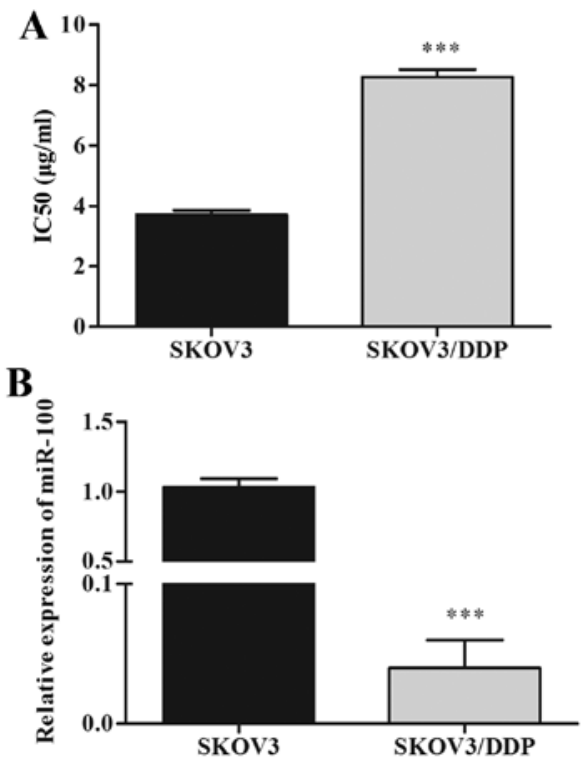

Figure $1 . \mathrm{IC}_{50}$ of ovary cancer cell lines SKOV3/DDP and SKOV3 to cisplatin (A) and relative expression of miR-100 in the cell lines (B). ${ }^{* * *} \mathrm{P}<0.001$.
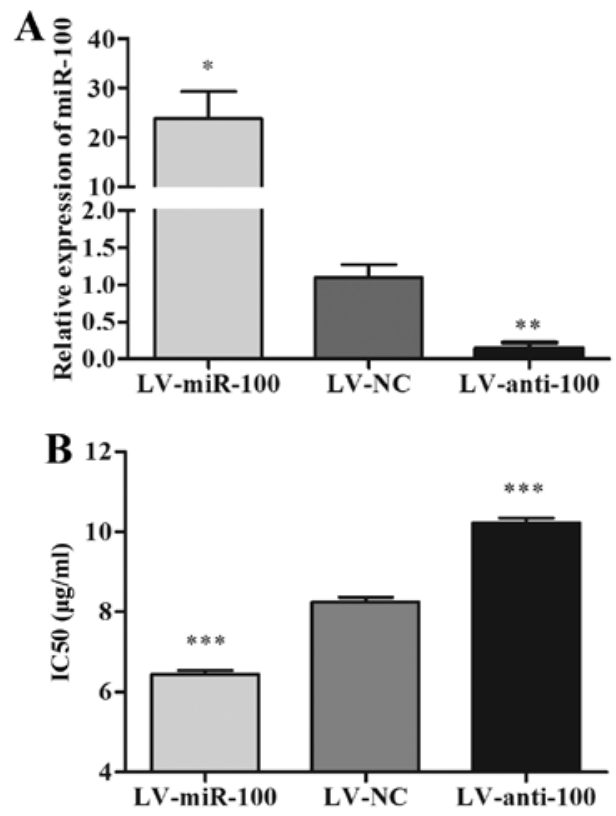

Figure 2. Relative miR-100 expression (A) and $\mathrm{IC}_{50}$ (B) of SKOV3/DDP cells transfected with miR-100 upregulation or downregulation constructs. ${ }^{*} \mathrm{P}<0.05,{ }^{* *} \mathrm{P}<0.01$ and ${ }^{* * *} \mathrm{P}<0.001$, respectively.

miR-100 was 25 times less in SKOV3/DDP cells as compared with SKOV3 cells (Fig. 1B). However, after transfection with the miR-100 mimics, the level of miR-100 in transfected SKOV3/DDP cells was significantly higher than that in LV-NC transfected cells $(\mathrm{P}<0.05$; Fig. $2 \mathrm{~A})$, while transfection with the miR-100 antisense resulted in significantly reduced miR-100 level in SKOV3/DDP cells ( $\mathrm{P}<0.01 ;$ Fig. 2A).

Change in $I C_{50}$ after transfection. The sensitivity of resistant SKOV3/DDP cells to cisplatin was determined after the cells were upregulated by miR-100 mimics. Compared with the cells transfected with LV-NC, cells transfected with LV-miR-100

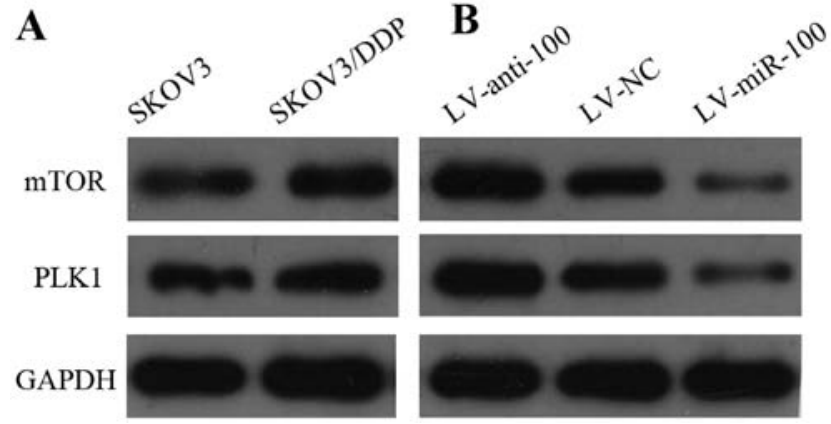

Figure 3. Expression of mTOR and PLK1 in SKOV3 and SKOV3/DDP cells before (A) and after (B) transfection with miR-100 upregulation or downregulation constructs revealed using western blot analysis.

had significantly low $\mathrm{IC}_{50}$ to cisplatin $(\mathrm{P}<0.001)$. On the other hand, when transfected with LV-anti-100, the $\mathrm{IC}_{50}$ was significantly increased ( $\mathrm{P}<0.001$; Fig. 2B).

Expression of mTOR and PLK1. Next, we examined the expression of mTOR and PLK1 in the cell lines before and after transfection using western blot analysis. Compared with SKOV 3 cells, mTOR and PLK1 protein levels were significantly higher in the resistant SKOV3/DDP cells $(\mathrm{P}<0.05$; Fig. 3A). After transfection with LV-miR-100, the levels of the two proteins were significantly lower than these in the LV-NCtransfected cells $(\mathrm{P}<0.05$; Fig. 3B), while transfection with LV-anti-100 resulted in significantly higher expression of the two proteins $(\mathrm{P}<0.05$; Fig. 3B).

Effect of miR-100 on the proliferation of SKOV3/DDP cells. The numbers of colony were reduced or increased after SKOV3/DDP cells were transfected with LV-miR-100 or LV-anti-100, respectively (Fig. 4A and B). After the transfection with LV-miR-100, the growth of SKOV3/DDP cells was reduced as revealed by the proliferation index (Fig. 4C); on the other hand, transfection with LV-anti-100 increased the proliferation index, as compared with LV-NC (Fig. 4C).

Effect of miR-100 on early apoptosis of SKOV3/DDP cells. After $48 \mathrm{~h}$ of cisplatin treatment at $3 \mu \mathrm{g} / \mathrm{ml}$, we examined the early apoptosis rate in SKOV3/DDP cells. The results showed that the apoptosis rate was significantly higher after the cells were transfected with LV-miR-100 as compared with LV-NC-transfected cells $(\mathrm{P}<0.01)$, while the apoptosis rate in SKOV3/DDP cells transfected with LV-anti-100 was significantly lower $(\mathrm{P}<0.05$; Fig. 5A and $\mathrm{C})$.

Effect of miR-100 on SKOV3/DDP cell cycle. The proportions of G1 and S phase cells in LV-miR-100- transfected cells was significantly higher or lower than those in LV-NC-transfected cells $(\mathrm{P}<0.01$; Fig. 5B and $\mathrm{D})$, respectively; in contrast, the proportions of $\mathrm{G} 1$ and $\mathrm{S}$ phase cells in $\mathrm{LV}$-anti-100-transfected cells were significantly lower or higher than those in LV-NCtransfected cells $(\mathrm{P}<0.05$; Fig. 5B and D), respectively.

Effect of miR-100 on efficacy of cisplatin in grafted resistant tumors in mice. We then used mouse models with grafted cells to assess the efficacy of cisplatin. When the mice were grafted 


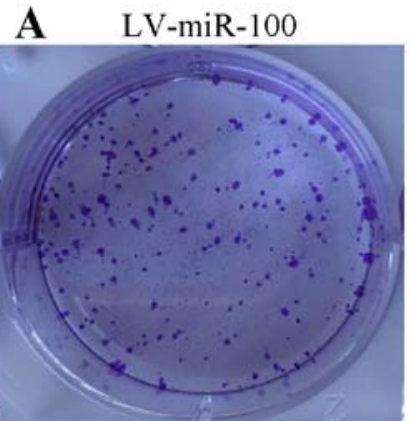

B

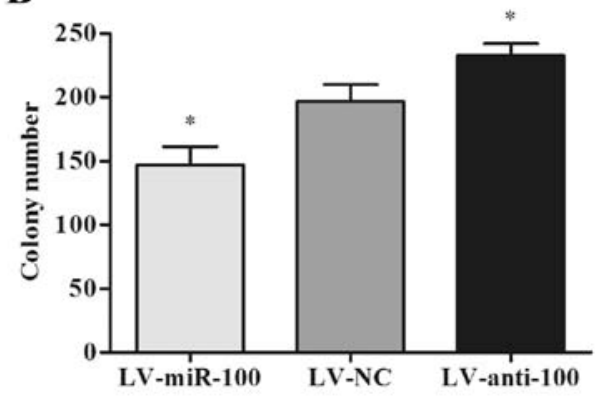

LV-NC

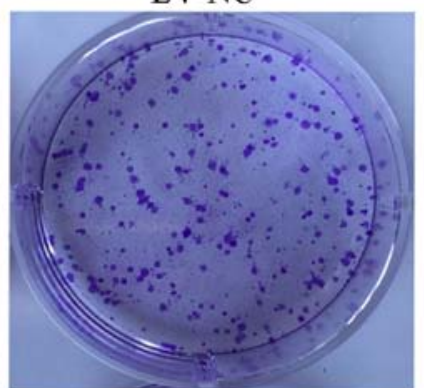

C

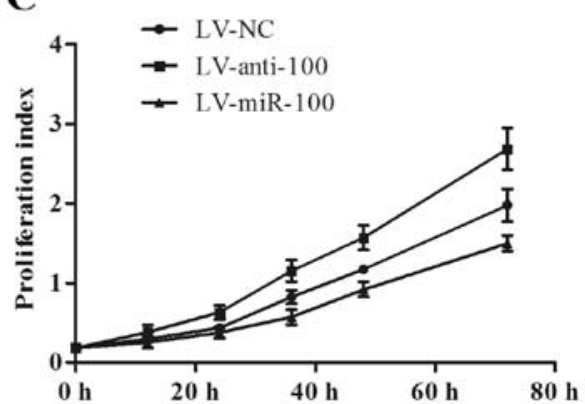

Figure 4. (A) Colonies, (B) their numbers and (C) growth curves of SKOV3/DDP transfected with miR-100 mimic and antisense constructs. "P<0.05 vs. LV-NC.

A
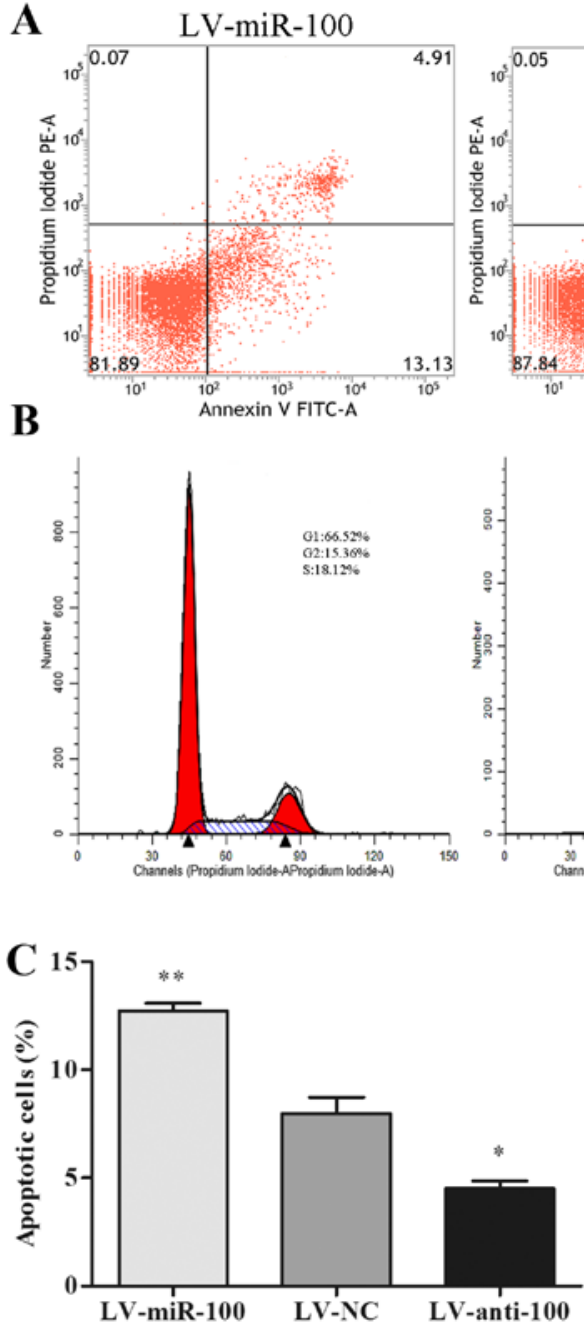

LV-NC
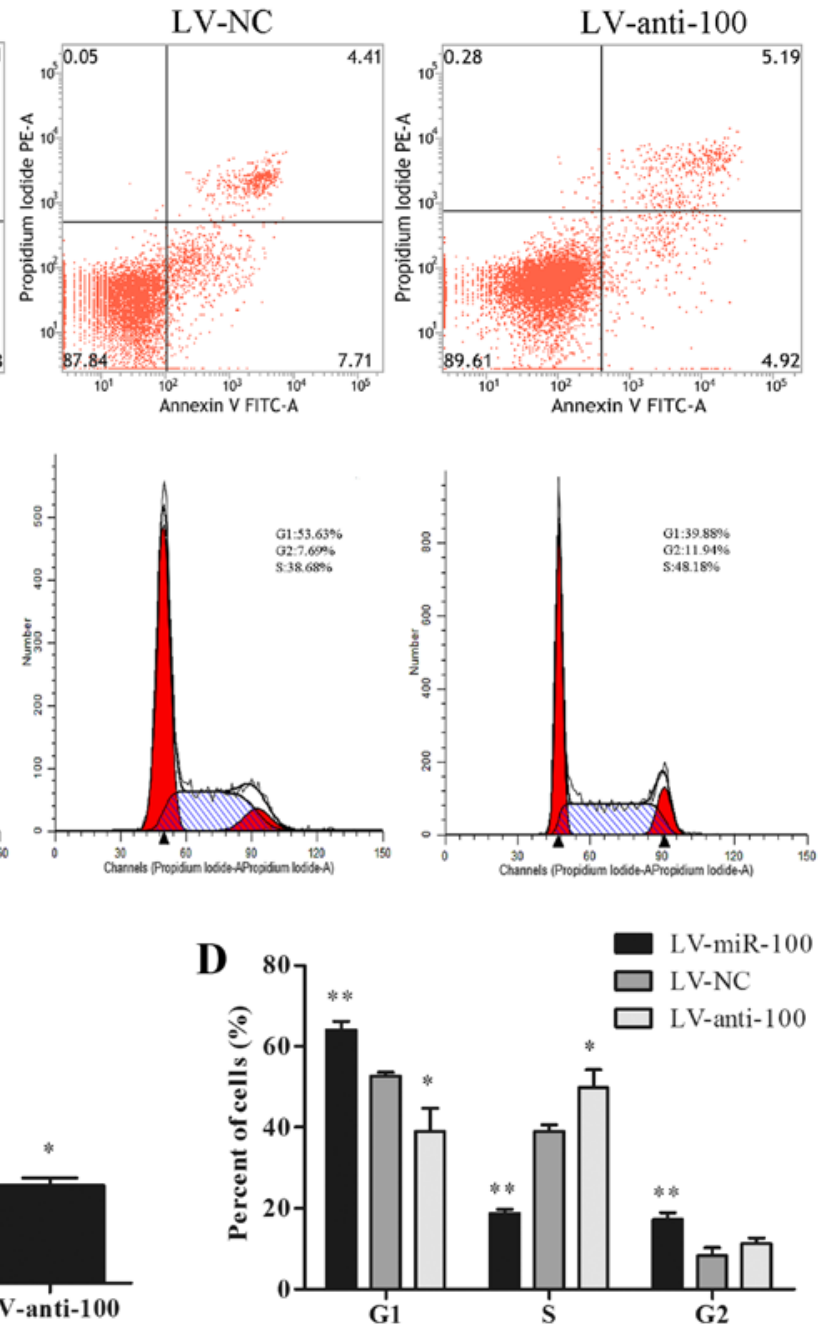

LV-anti-100
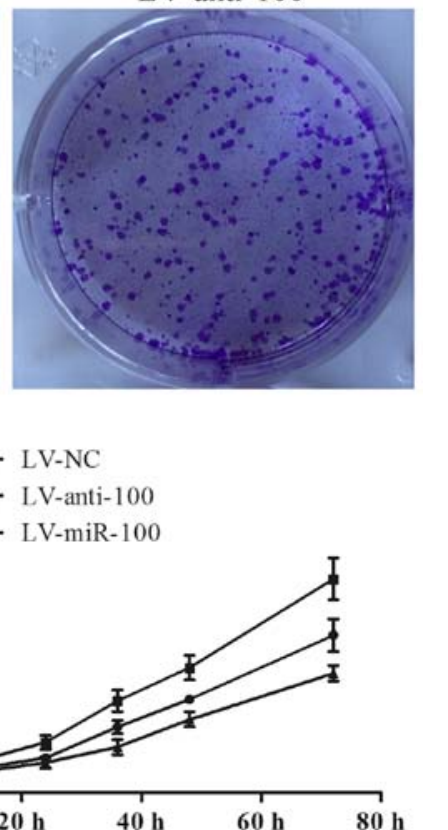
A

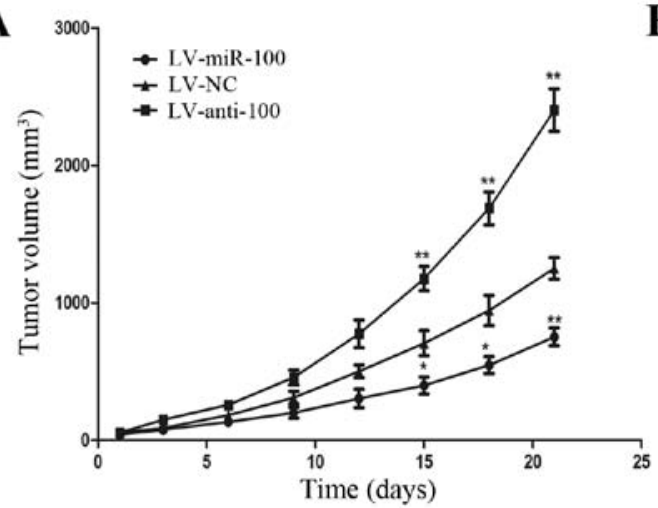

B

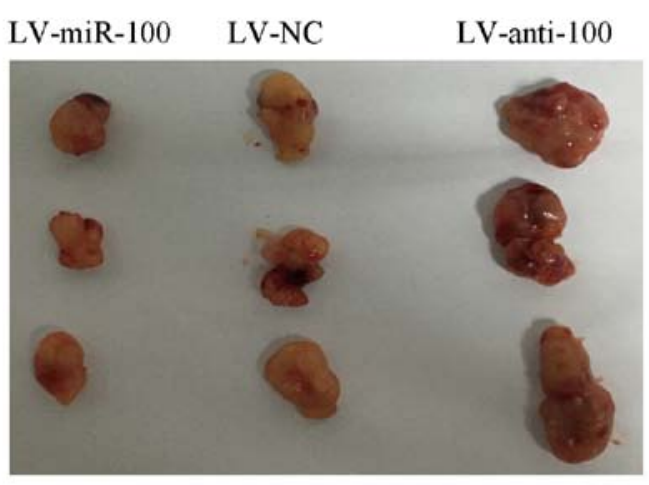

C
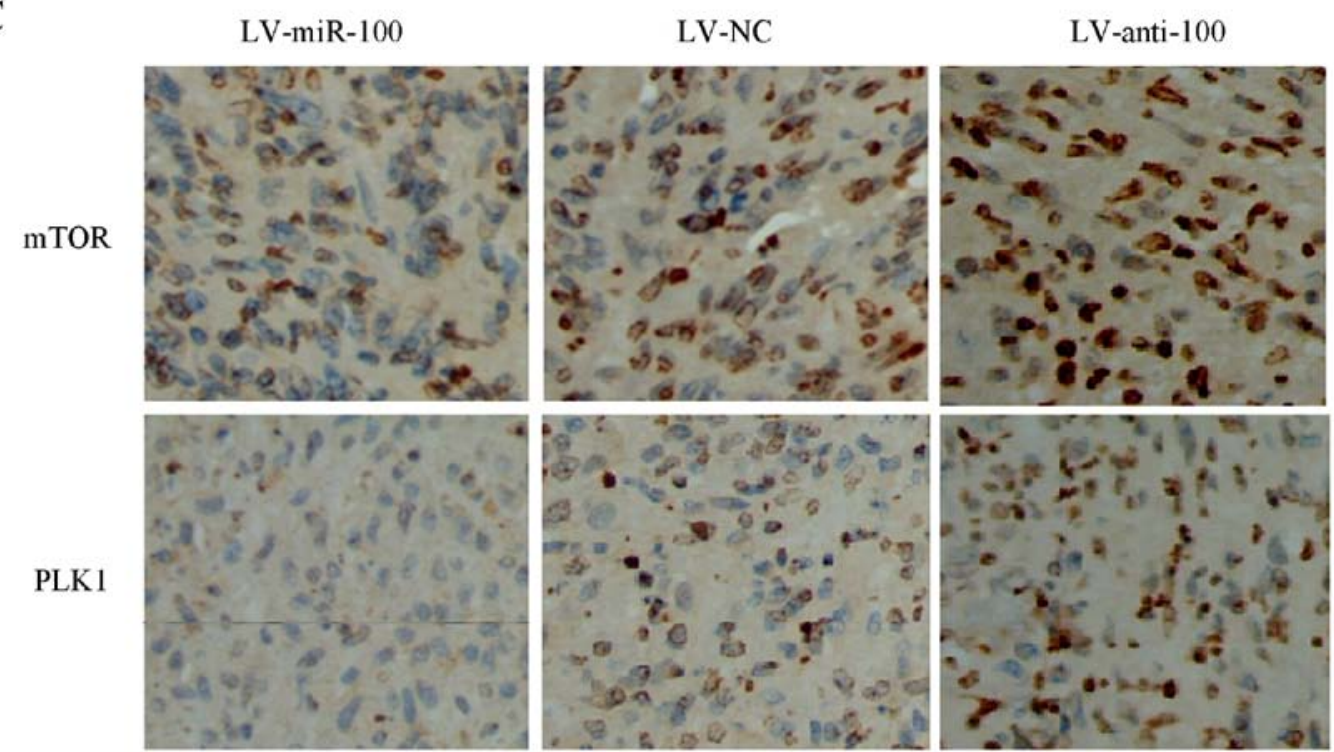

Figure 6. Growth (A) of tumors (at day 21, B) and expression (C) of mTOR and PLK1 in tumors derived from SKOV3/DDP cells stably transfected with miR-100 mimic and antisense constructs. ${ }^{*} \mathrm{P}<0.05$ or ${ }^{* *} \mathrm{P}<0.01$ vs. LV-NC.

with SKOV3/DDP cells transfected with LV-miR-100, the tumors grew slower than those grafted with LV-NC-transfected SKOV3/DDP cells. On other hand, tumors grew faster in mice grafted with LV-anti-100 than with LV-NC-transfected SKOV3/DDP cells (Fig. 6A). Twenty-one days after cisplatin treatment, the tumor volumes were smaller from SKOV3/DDP cells stably transfected with LV-miR-100 than with LV-NC (Fig. 6A and $\mathrm{B} ; \mathrm{P}<0.01$ ). In contrast, the tumors derived from SKOV3/DDP cells stably transfected with LV-anti-100 were significantly larger than with $\mathrm{LV}-\mathrm{NC}(\mathrm{P}<0.01$; Fig. $6 \mathrm{~A}$ and $\mathrm{B})$. Immunohistochemical analysis indicated that compared with tumors derived from LV-NC transfected cells, mTOR and PLK1 were downregulated in the tumors derived from LV-miR-100 transfected-cells and upregulated in the tumors derived from LV-anti-100 transfected cells $(\mathrm{P}<0.05$; Fig. 6C), respectively.

\section{Discussion}

Recent studies have shown that the abnormal expression of miRNAs is closely related to the occurrence, development, metastasis and prognosis of tumor, suggesting that miRNAs play a pivotal and regulatory role in the occurrence and development of tumors (15). Various miRNAs are demonstrated to impact the sensitivity of ovarian cancer to chemotherapy (16). For example, Chan et al (17) found that the suppression of miR-21 expression increases the chemosensitivity of ovarian cancer to cisplatin and paclitaxel and induces apoptosis of ovarian cancer cells. Wang et al (18) showed that the expression of miR-182 is associated with the tolerance of ovarian cancer cells to platinum-based drugs and paclitaxel and that suppression of miR-182 expression sensitizes ovarian cancer cells to these drugs. Abnormally expressed miRNAs in ovarian cancer may regulate the expression of genes that are responsible for drug tolerance, thereby influencing the outcome and prognosis of chemotherapy. Zhang et al (19) found that in ovarian cancer cell line A2780/DDP, upregulation of miR-182 expression results in targeted suppression of XIAP and restores the sensitivity of the cell line to platinum drugs; Cochrane et al (20) found that upregulation of $\mathrm{miR}-200 \mathrm{c}$ in ovarian cancer cell line Hey leads to targeted repression of TUBB3 and sensitizes the cell line to paclitaxel. These studies demonstrate that miRNAs may be used as a biomarker for the treatment of ovarian cancer and have potential in clinical applications and may serve as important targets for reversing drug resistance in ovarian cancer.

miR-100 was found early to be related to tumor. Studies have shown that it is lowly expressed in malignant tumors such 
as nasopharyngeal carcinoma, non-small cell lung, bladder cancer, hepatocellular carcinoma and ovarian cancer (21-26), where it functions as tumor repressor. Studies also show that it is possible to regulate the chemosensitivity and prognosis of cancer by targeted regulation of expression of genes involved in drug sensitivity (27-29). In our earlier study, we found that there is an association between the expression of miR-100 in EOC patients and clinical or pathological outcomes. We found that miR-100 is negatively correlated with the clinical stage, metastasis, relapse and prognosis of ovarian cancer after chemotherapy (30). In this study, we show that the expression level of miR-100 was lower in drug tolerant SKOV3/DDP cells than in their parent cells, suggesting that miR-100 may be associated with cisplatin resistance in EOC. Upregulation of miR-100 in SKOV3/DDP cells with miR-100 mimics reduced the $\mathrm{IC}_{50}$ of the tolerant cells to cisplatin, while downregulation of miR-100 with antisense miR-100 increased the $\mathrm{IC}_{50}$. These results indicate that the expression level of miR-100 is a factor affecting the sensitivity of EOC cells to cisplatin. We then investigated the mechanism underlying the regulation of cisplatin sensitivity by miR-100. Our data showed that miR-100 increases the sensitivity probably by inhibiting cell proliferation, promoting cell apoptosis and cell cycle arrest.

Previous studies have indicated that mTOR and PLK1 are the target molecules of miR-100 (31,32). mTOR and PLK1 are members of highly conserved serine/threonine kinase family, which are widely present in eukaryotic cells, and are involved in cell proliferation, metabolism, metastasis and other biological processes (33). PLK1 and PLK1 are highly expressed in many human cancers, including ovarian cancer processes (34), and play an important role in regulating the growth and metastasis of tumor cells and in determining the sensitivity to chemotherapy $(35,36)$. Yang et al (37) showed that the mTOR pathway is an intracellular signaling pathway important in regulating the sensitivity of ovarian cancer cells to cisplatin. Gao et al (38) suggested that PLK1 is an independent prognostic factor for ovarian cancer and silencing PLK1 increases the sensitivity of ovarian cancer cells to cisplatin chemotherapy. In this study, the expression of mTOR and PLK1 was significantly higher in SKOV3/DDP cells than in SKOV3 cells, suggesting that the overexpression of the mTOR and PLK1 genes may be associated with cisplatin resistance in EOC. Once miR-100 expression was upregulated, mTOR and PLK1 expression was decreased, vice versa. These findings demonstrate that miR-100 may sensitize EOC to cisplatin by targeted downregulation of mTOR and PLK1 expression.

In summary, the present study demonstrates that upregulation of miR-100 can inhibit cell proliferation, promote cell apoptosis and cell cycle arrest, and downregulate mTOR and PLK1 expression in EOC; as a result, it sensitize the resistant EOC cells to cisplatin, resulting in reversed drug resistance. These findings provide new insights into the mechanism of drug resistance in EOC, solution to overcome the drug resistance and help to identify new targets for the gene therapy of drug resistant ovarian cancer.

\section{Acknowledgements}

The present study was supported by the Science and Technology Funds of Guangdong Province (2013B021800303).

\section{References}

1. Kuhlmann JD, Rasch J, Wimberger P and Kasimir-Bauer S: microRNA and the pathogenesis of ovarian cancer - a new horizon for molecular diagnostics and treatment? Clin Chem Lab Med 50: 601-615, 2012.

2. Siegel R, Ma J, Zou Z and Jemal A: Cancer statistics, 2014. CA Cancer J Clin 64: 9-29, 2014.

3. Yakirevich E, Sabo E, Naroditsky I, Sova Y, Lavie O and Resnick MB: Multidrug resistance-related phenotype and apoptosis-related protein expression in ovarian serous carcinomas. Gynecol Oncol 100: 152-159, 2006.

4. Wenham RM, Lapolla J, Lin HY, Apte SM, Lancaster JM, Judson PL, Gonzalez-Bosquet J, Herschberger A, Havrilesky LJ and Secord AA: A phase II trial of docetaxel and bevacizumab in recurrent ovarian cancer within 12 months of prior platinumbased chemotherapy. Gynecol Oncol 130: 19-24, 2013.

5. Shen DW, Pouliot LM, Hall MD and Gottesman MM: Cisplatin resistance: A cellular self-defense mechanism resulting from multiple epigenetic and genetic changes. Pharmacol Rev 64: 706-721, 2012.

6. Morin PJ: Drug resistance and the microenvironment: Nature and nurture. Drug Resist Updat 6: 169-172, 2003.

7. Brabec V and Kasparkova J: Molecular aspects of resistance to antitumor platinum drugs. Drug Resist Updat 5: 147-161, 2002.

8. Hong L, Yang Z, Ma J and Fan D: Function of miRNA in controlling drug resistance of human cancers. Curr Drug Targets 14: 1118-1127, 2013.

9. Sorrentino A, Liu CG, Addario A, Peschle C, Scambia G and Ferlini C: Role of microRNAs in drug-resistant ovarian cancer cells. Gynecol Oncol 111: 478-486, 2008.

10. Bartel DP: MicroRNAs: Genomics, biogenesis, mechanism, and function. Cell 116: 281-297, 2004.

11. Barbarotto E, Schmittgen TD and Calin GA: MicroRNAs and cancer: Profile, profile, profile. Int J Cancer 122: 969-977, 2008.

12. Nagaraja AK, Creighton CJ, Yu Z, Zhu H, Gunaratne PH, Reid JG, Olokpa E, Itamochi H, Ueno NT, Hawkins SM, et al: A link between mir-100 and FRAP1/mTOR in clear cell ovarian cancer. Mol Endocrinol 24: 447-463, 2010.

13. Feng B, Wang R and Chen L-B: MiR-100 resensitizes docetaxelresistant human lung adenocarcinoma cells (SPC-A1) to docetaxel by targeting Plk1. Cancer Lett 317: 184-191, 2012.

14. Schmittgen TD and Livak KJ: Analyzing real-time PCR data by the comparative C(T) method. Nat Protoc 3: 1101-1108, 2008

15. Yang W, Lee DY and Ben-David Y: The roles of microRNAs in tumorigenesis and angiogenesis. Int $\mathrm{J}$ Physiol Pathophysiol Pharmacol 3: 140-155, 2011.

16. Sarkar FH, Li Y, Wang Z, Kong D and Ali S: Implication of microRNAs in drug resistance for designing novel cancer therapy. Drug Resist Updat 13: 57-66, 2010.

17. Chan JK, Blansit K, Kiet T, Sherman A, Wong G, Earle C and Bourguignon LY: The inhibition of miR-21 promotes apoptosis and chemosensitivity in ovarian cancer. Gynecol Oncol 132: 739-744, 2014.

18. Wang YQ, Guo RD, Guo RM, Sheng W and Yin LR: MicroRNA182 promotes cell growth, invasion, and chemoresistance by targeting programmed cell death 4 (PDCD4) in human ovarian carcinomas. J Cell Biochem 114: 1464-1473, 2013.

19. Zhang X, Huang L, Zhao Y and Tan W: Downregulation of miR-130a contributes to cisplatin resistance in ovarian cancer cells by targeting X-linked inhibitor of apoptosis (XIAP) directly. Acta Biochim Biophys Sin (Shanghai) 45: 995-1001, 2013.

20. Cochrane DR, Howe EN, Spoelstra NS and Richer JK: Loss of miR-200c: A marker of aggressiveness and chemoresistance in female reproductive cancers. J Oncol 2010: 821717, 2010. doi: 10.1155/2010/821717.

21. Shi W, Alajez NM, Bastianutto C, Hui AB, Mocanu JD, Ito E, Busson P, Lo KW, Ng R, Waldron J, et al: Significance of Plk1 regulation by miR-100 in human nasopharyngeal cancer. Int J Cancer 126: 2036-2048, 2010.

22. Liu J, Lu KH, Liu ZL, Sun M, De W and Wang ZX: MicroRNA100 is a potential molecular marker of non-small cell lung cancer and functions as a tumor suppressor by targeting polo-like kinase 1. BMC Cancer 12: 519, 2012.

23. Wang S, Xue S, Dai Y, Yang J, Chen Z, Fang X, Zhou W, Wu W and Li Q: Reduced expression of microRNA-100 confers unfavorable prognosis in patients with bladder cancer. Diagn Pathol 7: 159, 2012. 
24. Chen P, Zhao X and Ma L: Downregulation of microRNA-100 correlates with tumor progression and poor prognosis in hepatocellular carcinoma. Mol Cell Biochem 383: 49-58, 2013.

25. Yang H, Kong W, He L, Zhao JJ, O'Donnell JD, Wang J, Wenham RM, Coppola D, Kruk PA, Nicosia SV, et al: MicroRNA expression profiling in human ovarian cancer: miR-214 induces cell survival and cisplatin resistance by targeting PTEN. Cancer Res 68: 425-433, 2008.

26. Wyman SK, Parkin RK, Mitchell PS, Fritz BR, O'Briant K, Godwin AK, Urban N, Drescher CW, Knudsen BS and Tewari M: Repertoire of microRNAs in epithelial ovarian cancer as determined by next generation sequencing of small RNA cDNA libraries. PLoS One 4: e5311, 2009.

27. Blick C, Ramachandran A, Wigfield S, McCormick R, Jubb A, Buffa FM, Turley H, Knowles MA, Cranston D, Catto J, et al: Hypoxia regulates FGFR3 expression via HIF-1 $\alpha$ and miR-100 and contributes to cell survival in non-muscle invasive bladder cancer. Br J Cancer 109: 50-59, 2013.

28. Xiao F, Bai Y, Chen Z, Li Y, Luo L, Huang J, Yang J, Liao H and Guo L: Downregulation of HOXA1 gene affects small cell lung cancer cell survival and chemoresistance under the regulation of miR-100. Eur J Cancer 50: 1541-1554, 2014.

29. Gebeshuber CA and Martinez J: miR-100 suppresses IGF2 and inhibits breast tumorigenesis by interfering with proliferation and survival signaling. Oncogene 32: 3306-3310, 2013.

30. Peng DX, Luo M, Qiu LW, He YL and Wang XF: Prognostic implications of microRNA-100 and its functional roles in human epithelial ovarian cancer. Oncol Rep 27: 1238-1244, 2012.
31. Zhu Z, Wang CP, Zhang YF and Nie L: MicroRNA-100 resensitizes resistant chondrosarcoma cells to cisplatin through direct targeting of mTOR. Asian Pac J Cancer Prev 15: 917-923, 2014.

32. Li BH, Zhou JS, Ye F, Cheng XD, Zhou CY, Lu WG and Xie X: Reduced miR-100 expression in cervical cancer and precursors and its carcinogenic effect through targeting PLK1 protein. Eur J Cancer 47: 2166-2174, 2011.

33. Degenhardt $\mathrm{Y}$ and Lampkin T: Targeting Polo-like kinase in cancer therapy. Clin Cancer Res 16: 384-389, 2010.

34. Fruman DA and Rommel C: PI3K and cancer: Lessons, challenges and opportunities. Nat Rev Drug Discov 13: 140-156, 2014.

35. Takaki T, Trenz K, Costanzo V and Petronczki M: Polo-like kinase 1 reaches beyond mitosis - cytokinesis, DNA damage response, and development. Curr Opin Cell Biol 20: 650-660, 2008.

36. Weichert W, Denkert C, Schmidt M, Gekeler V, Wolf G, Köbel M, Dietel $\mathrm{M}$ and Hauptmann S: Polo-like kinase isoform expression is a prognostic factor in ovarian carcinoma. $\mathrm{Br} \mathrm{J}$ Cancer 90: 815-821, 2004.

37. Yang X, Fraser M, Abedini MR, Bai T and Tsang BK: Regulation of apoptosis-inducing factor-mediated, cisplatin-induced apoptosis by Akt. Br J Cancer 98: 803-808, 2008.

38. Gao Q, Huang X, Tang D, Cao Y, Chen G, Lu Y, Zhuang L, Wang S, Xu G, Zhou J, et al: Influence of chk1 and plk1 silencing on radiation- or cisplatin-induced cytotoxicity in human malignant cells. Apoptosis 11: 1789-1800, 2006. 